\title{
Observability of a ring shaped membrane via Fourier series
}

\author{
Vilmos Komornik, Paola Loreti, and Michel Mehrenberger \\ Université de Strasbourg, 7 rue René Descartes, F-67084 Strasbourg \\ komornik@math.unistra.fr \\ Dipartimento di Scienze di Base e Applicate per l'Ingegneria, \\ Sapienza Università di Roma, Via A. Scarpa 16, I-00161 Roma \\ paola.loreti@sbai.uniroma1.it \\ Université de Strasbourg, 7 rue René Descartes, F-67084 Strasbourg \\ mehrenbe@math.unistra.fr
}

\begin{abstract}
We study the inverse Ingham type inequality for a wave equation in a ring. This leads to a conjecture on the zeros of Bessel cross product functions. We motivate the validity of the conjecture through numerical results. We do a complete analysis in the particular case of radial initial data, where an improved time of observability is available.
\end{abstract}

\section{Introduction}

We study the solution $u=u(t, r, \theta)$ of the wave equation in an annulus $\Omega$ of small radius $a$ and big radius $b$ :

$$
\left\{\begin{array}{l}
u^{\prime \prime}=\Delta u, \quad 0<t<T, \quad x \in \Omega \\
u=0, \quad 0<t<T, \quad x \in \Gamma=\partial \Omega \\
u(0, x)=u_{0}(x), \quad \partial_{t} u(0, x)=u_{1}(x), \quad x \in \Omega .
\end{array}\right.
$$

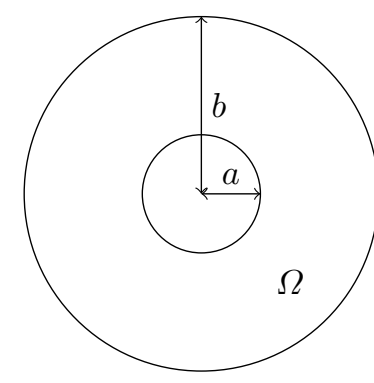

We have the following classical observability estimate (see [7] for example):

Proposition 1. There exists $T_{0}>0$ such that for $T>T_{0}$ the system (1) is observable: there exists a constant $c>0$ such that we have

$$
\left\|u_{0}\right\|_{H_{0}^{1}(\Omega)}^{2}+\left\|u_{1}\right\|_{L^{2}(\Omega)}^{2} \leq c \int_{0}^{T} \int_{\Gamma}\left|\partial_{\nu} u(t, x)\right|^{2} \mathrm{~d} \Gamma \mathrm{d} t,
$$

for all $\left(u_{0}, u_{1}\right) \in H_{0}^{1}(\Omega) \times L^{2}(\Omega)$. 
This proposition is a special case of a general result, which can be proved by micro-local analysis [2] with the critical time $T_{0}=2 \sqrt{b^{2}-a^{2}}$, according to the geometrical ray condition.

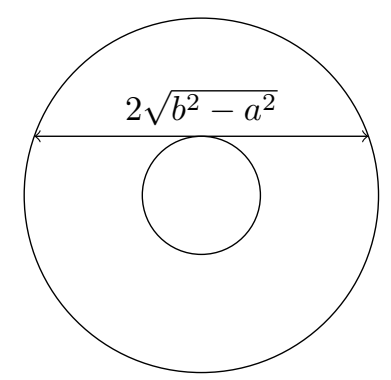

Our aim here is to study this problem using a Fourier series approach [6,5] Note that such an approach has been tackled for the whole disk (see [7]); the case of an annulus leads to new difficulties. In particular, we are not able to treat the general case, as it relies on very precise estimates of the location of the zeros of Bessel cross product functions, that we did not find in the literature. Instead, we state a conjecture on these zeros and give some numerical results to support the conjecture. In the case of radial functions or more generally functions with a limited number of modes in the angle direction, we are able to get the observability estimates with this method, even for smaller times $T>2(b-a)$, using an asymptotic result of MacMahon (see [1], p. 374).

In Section 2, we give the expression of the solution. In Section 3, we formulate a theorem for the special case of radial functions. Section 4 is devoted to the statement of the conjecture and its numerical illustration. Finally, we prove in Section 5 the theorem of Section 3 .

\section{Expression of the solution}

Let $J_{\nu}$ (resp. $Y_{\nu}$ ) be the Bessel functions of first (resp. second) kind of order $\nu$. We recall the following proposition (see $[7,8]$ )

Proposition 2. (i) Let $\nu \in \mathbb{R}^{*}$ and $0<\alpha<1$. The nonnegative zeros $\gamma_{\nu, k}, k \in$ $\mathbb{N}^{*}$ of the Bessel cross product function

$$
H_{\nu, \alpha}(x)=Y_{\nu}(x) J_{\nu}(\alpha x)-J_{\nu}(x) Y_{\nu}(\alpha x),
$$

form a strictly increasing sequence:

$$
0<\gamma_{\nu, \alpha, 1}<\gamma_{\nu, \alpha, 2}<\ldots \gamma_{\nu, \alpha, k}<\ldots, k \in \mathbb{N}^{*} .
$$

(ii) The eigenfunctions of the Laplacian corresponding to (1) are $R_{k, m}(r) e^{i m \theta}$ for $k \in \mathbb{N}^{*}$ and $m \in \mathbb{N}$, where

$$
R_{k, m}(r)=Y_{m}\left(\gamma_{m, \alpha, k}\right) J_{m}\left(\frac{r}{b} \gamma_{m, \alpha, k}\right)-J_{m}\left(\gamma_{m, \alpha, k}\right) Y_{m}\left(\frac{r}{b} \gamma_{m, \alpha, k}\right)
$$


(iii) For a dense set of initial data the solution of (1) is given by the formula

$$
u(t, r, \theta)=\sum_{m=0}^{\infty} \sum_{k=1}^{\infty} R_{k, m}(r)\left(c_{k, m}^{+} e^{i m \theta} e^{i \frac{\gamma_{m, \alpha, k}}{b} t}+c_{k, m}^{-} e^{-i m \theta} e^{-i \frac{\gamma_{m, \alpha, k}}{b} t}\right)
$$

with complex coefficients $c_{k, m}^{ \pm}$, all but finitely of which vanish.

\section{Observability estimates for radial solutions}

We recall from [1] the following estimate of MacMahon (1894):

$$
\gamma_{\nu, \alpha, k}=\frac{\pi k}{1-\alpha}+\frac{4 \nu^{2}-1}{8 \pi \alpha} \frac{1-\alpha}{k}+O\left(\frac{1-\alpha}{k}\right)^{3} .
$$

Thanks to this estimation, we can obtain the following theorem.

Theorem 1. Let $T>2(b-a)$. For each positive integer $M$ there exists a constant $c_{M}>0$ such that

$$
\left\|u_{0}\right\|_{H_{0}^{1}(\Omega)}^{2}+\left\|u_{1}\right\|_{L^{2}(\Omega)}^{2} \leq c_{M} \int_{0}^{T} \int_{\Gamma}\left|\partial_{\nu} u(t, x)\right|^{2} \mathrm{~d} \Gamma \mathrm{d} t,
$$

for all solutions of (1) of the form (4) with $c_{k, m}^{ \pm}=0$ whenever $m \geq M$.

Remark 1. - This theorem covers the case of radial initial data, corresponding to the case $M=1$.

- If $2 \sqrt{b^{2}-a^{2}}>T>2(b-a)$, then the constant $c_{M}$ tends to zero as $M \rightarrow \infty$ because $T_{0}=2 \sqrt{b^{2}-a^{2}}$ is the critical observability time for general initial data.

- The theorem remains true if the integral in (6) is taken only over the outer boundary (the circle of radius $b$ ): see the estimate (16)) below.

- We may obtain similar results by changing the boundary conditions. For example, we may take homogeneous Neumann condition on the inner boundary, and observe the solution only on the outer boundary (see [4] for the corresponding asymptotic gap estimate that is needed).

\section{Conjecture and numerical illustration}

We then state the following conjecture.

Conjecture 1 Let $0<\alpha<1$ and $\nu>1 / 2$.

- There exists a positive integer $k_{\nu}(\alpha)$ such that $\gamma_{\nu, \alpha, k+1}-\gamma_{\nu, \alpha, k}$ is decreasing for $k \leq k_{\nu}(\alpha)$ and increasing for $k \geq k_{\nu}(\alpha)$.

- We have $\gamma_{\nu, \alpha, k_{\nu}(\alpha)+1}-\gamma_{\nu, \alpha, k_{\nu}(\alpha)} \geq \pi\left(1-\alpha^{2}\right)^{-1 / 2}$. 
Using a Bessel Zeros Computer [3] we can evaluate the zeros $\gamma_{\nu, \alpha, k}$ for several parameters $\nu, \alpha$ and $k$.

We plot on Figure 1 (top and bottom left) the values $k_{\nu}(\alpha)$ versus $\nu$ for different values of $\alpha$. We observe that $k_{\nu}(\alpha)$ increases with $\nu$ for a fixed $\alpha$. The dependance seems to be almost linear, except for small values of $\alpha$ where the dependence seems to be quadratic (see Figure 1, bottom left). On Figure 1 (bottom right), we see the relative difference with the gap $\frac{\pi}{\sqrt{1-\alpha^{2}}}$ (corresponding to the optimal value of Proposition 1). We observe that $\gamma_{\nu, \alpha, k_{\nu}(\alpha)+1}-\gamma_{\nu, \alpha, k_{\nu}(\alpha)}$ decreases and approaches to this gap as $\nu$ increases.
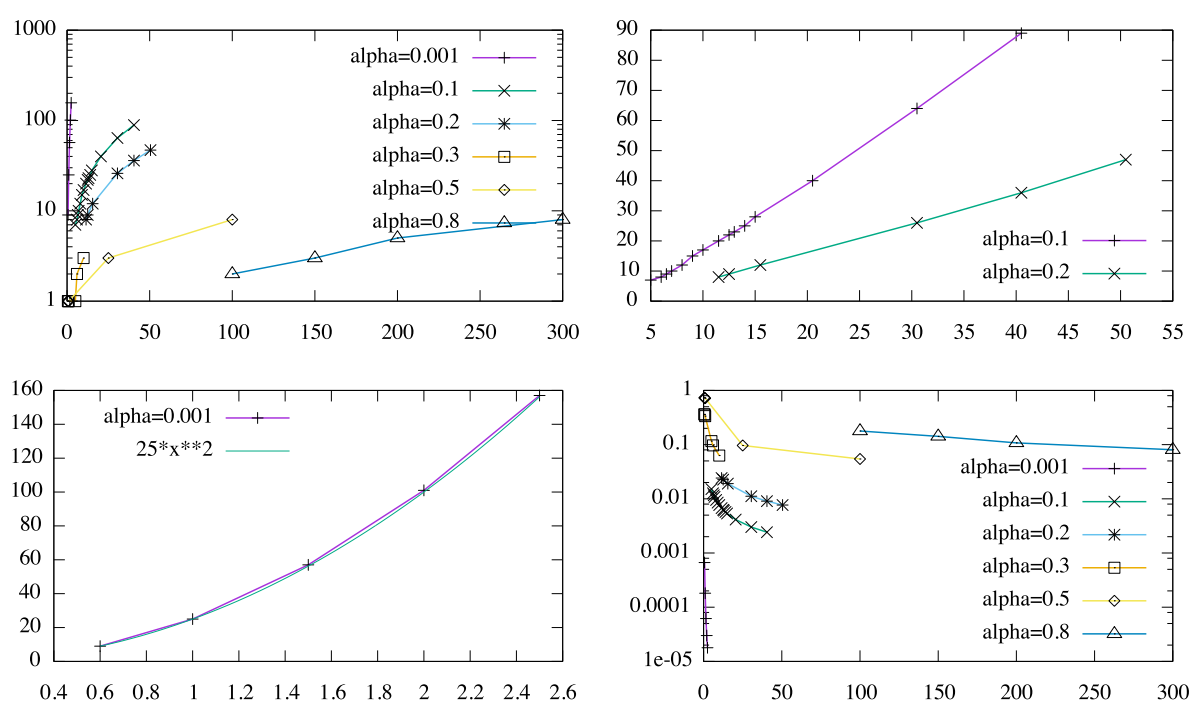

Fig. 1. Index $k_{\nu}(\alpha)$ where minimal gap occurs versus $\nu \geq 1 / 2$ (top left, logarithmic plot for $\alpha \in\{0.001,0.1,0.2,0.3,0.5,0.8\}$; top right, standard plot for $\alpha=0.1$ and $\alpha=0.2$; bottom left, $\alpha=0.001$ and comparison with function $\left.0.25 \nu^{2}\right)$. Difference $\frac{\gamma_{\nu, \alpha}, k_{\nu}(\alpha)+1-\gamma_{\nu, \alpha, k_{\nu}(\alpha)}}{\pi / \sqrt{1-\alpha^{2}}}-1$ versus $\nu$ in logarithmic scale (bottom right).

\section{Proof of Theorem 1}

We first express the norm in terms of the Fourier coefficients. We have

$$
u(t, r, \theta)=\sum_{m=0}^{\infty} \sum_{k=1}^{\infty} R_{k, m}(r)\left(c_{k, m}^{+} e^{i m \theta} e^{i \frac{\gamma_{m, \alpha, k}}{b} t}+c_{k, m}^{-} e^{-i m \theta} e^{-i \frac{\gamma_{m, \alpha, k}}{b} t}\right)
$$

with

$$
R_{k, m}(r)=Y_{m}\left(\gamma_{m, \alpha, k}\right) J_{m}\left(\frac{r}{b} \gamma_{m, \alpha, k}\right)-J_{m}\left(\gamma_{m, \alpha, k}\right) Y_{m}\left(\frac{r}{b} \gamma_{m, \alpha, k}\right)
$$


This leads to the equalities

$$
u_{0}(r, \theta)=u(0, r, \theta)=\sum_{m=0}^{\infty} \sum_{k=1}^{\infty} R_{k, m}(r)\left(c_{k, m}^{+} e^{i m \theta}+c_{k, m}^{-} e^{-i m \theta}\right) .
$$

and

$$
u_{1}(r, \theta)=\partial_{t} u(0, r, \theta)=\sum_{m=0}^{\infty} \sum_{k=1}^{\infty} \frac{i \gamma_{m, \alpha, k}}{b} R_{k, m}(r) e^{i m \theta}\left(c_{k, m}^{+} e^{i m \theta}-c_{k, m}^{-} e^{-i m \theta}\right)
$$

Now, using the orthogonality of the eigenvectors of the Laplacian operator we obtain

$$
\begin{aligned}
& \left\|u_{1}\right\|_{L^{2}(\Omega)}^{2}=\int_{r=a}^{b} \int_{\theta=0}^{2 \pi}\left|u_{1}(r, \theta)\right|^{2} r d r d \theta \\
& =2 \pi \sum_{m=1}^{\infty} \sum_{k=1}^{\infty}\left|\frac{\gamma_{m, \alpha, k}}{b}\right|^{2}\left(\left|c_{k, m}^{+}\right|^{2}+\left|c_{k, m}^{-}\right|^{2}\right) \int_{a}^{b}\left|R_{k, m}(r)\right|^{2} r d r \\
& \quad+2 \pi \sum_{k=1}^{\infty}\left|\frac{\gamma_{0, \alpha, k}}{b}\right|^{2}\left|c_{k, 0}^{+}-c_{k, 0}^{-}\right|^{2} \int_{a}^{b}\left|R_{k, 0}(r)\right|^{2} r d r
\end{aligned}
$$

and a similar computation gives

$$
\begin{aligned}
\int_{\Omega}\left|u_{0}\right|^{2} d \Omega=2 \pi \sum_{m=1}^{\infty} \sum_{k=1}^{\infty}\left(\left|c_{k, m}^{+}\right|^{2}\right. & \left.+\left|c_{k, m}^{-}\right|^{2}\right) \int_{a}^{b}\left|R_{k, m}(r)\right|^{2} r d r \\
& +2 \pi \sum_{k=1}^{\infty}\left(\left|c_{k, 0}^{+}+c_{k, 0}^{-}\right|^{2}\right) \int_{a}^{b}\left|R_{k, 0}(r)\right|^{2} r d r
\end{aligned}
$$

Since

$$
\left\|u_{0}\right\|_{H_{0}^{1}(\Omega)}^{2}=\int_{\Omega}\left|\nabla u_{0}\right|^{2} d \Omega+\int_{\Omega}\left|u_{0}\right|^{2} d \Omega
$$

we have to compute also the the first integral on the right side. We have

$$
\int_{\Omega}\left|\nabla u_{0}\right|^{2} d \Omega=\int_{r=a}^{b} \int_{\theta=0}^{2 \pi}\left|\partial_{r} u_{0}\right|^{2} r d r d \theta+\int_{r=a}^{b} \int_{\theta=0}^{2 \pi} \frac{1}{r}\left|\partial_{\theta} u_{0}\right|^{2} d r d \theta .
$$

Since

$$
\partial_{r} u_{0}(r, \theta)=\partial_{r} u(0, r, \theta)=\sum_{m=0}^{\infty} \sum_{k=1}^{\infty} R_{k, m}^{\prime}(r)\left(c_{k, m}^{+} e^{i m \theta}+c_{k, m}^{-} e^{-i m \theta}\right)
$$

and

$$
\partial_{\theta} u_{0}(r, \theta)=\partial_{\theta} u(0, r, \theta)=\sum_{m=0}^{\infty} \sum_{k=1}^{\infty} i m R_{k, m}(r)\left(c_{k, m}^{+} e^{i m \theta}-c_{k, m}^{-} e^{-i m \theta}\right),
$$


using the orthogonality of the eigenfunctions, we get

$$
\begin{array}{r}
\int_{r=a}^{b} \int_{\theta=0}^{2 \pi}\left|\partial_{r} u_{0}\right|^{2} r d r d \theta=2 \pi \sum_{m=1}^{\infty} \sum_{k=1}^{\infty}\left(\left|c_{k, m}^{+}\right|^{2}+\left|c_{k, m}^{-}\right|^{2}\right) \int_{a}^{b}\left|R_{k, m}^{\prime}(r)\right|^{2} r d r, \\
+2 \pi \sum_{k=1}^{\infty}\left(\left|c_{k, 0}^{+}+c_{k, 0}^{-}\right|^{2}\right) \int_{a}^{b}\left|R_{k, 0}^{\prime}(r)\right|^{2} r d r
\end{array}
$$

and

$$
\int_{r=a}^{b} \int_{\theta=0}^{2 \pi} \frac{1}{r}\left|\partial_{\theta} u_{0}\right|^{2} d r d \theta=2 \pi \sum_{m=1}^{\infty} \sum_{k=1}^{\infty}\left(\left|c_{k, m}^{+}\right|^{2}+\left|c_{k, m}^{-}\right|^{2}\right) m^{2} \int_{a}^{b} \frac{1}{r}\left|R_{k, m}(r)\right|^{2} d r
$$

Using all these results we obtain the equalities

$$
\begin{array}{r}
E_{0}:=\left\|u_{0}\right\|_{H_{0}^{1}(\Omega)}^{2}+\left\|u_{1}\right\|_{L^{2}(\Omega)}^{2}=2 \pi \sum_{m=1}^{\infty} \sum_{k=1}^{\infty}\left(\left|c_{k, m}^{+}\right|^{2}+\left|c_{k, m}^{-}\right|^{2}\right) \\
\quad\left(\int_{a}^{b}\left(1+\left|\frac{\gamma_{m, \alpha, k}}{b}\right|^{2}+\frac{m^{2}}{r^{2}}\right)\left|R_{k, m}(r)\right|^{2} r d r+\int_{a}^{b}\left|R_{k, m}^{\prime}(r)\right|^{2} r d r\right) \\
+2 \pi \sum_{k=1}^{\infty}\left(\int_{a}^{b}\left(\left|c_{k, 0}^{+}+c_{k, 0}^{-}\right|^{2}+\left|c_{k, 0}^{+}-c_{k, 0}^{-}\right|^{2}\left|\frac{\gamma_{0, \alpha, k}}{b}\right|^{2}\right)\left|R_{k, 0}(r)\right|^{2} r d r\right. \\
\left.+\left|c_{k, 0}^{+}+c_{k, 0}^{-}\right|^{2} \int_{a}^{b}\left|R_{k, 0}^{\prime}(r)\right|^{2} r d r\right)
\end{array}
$$

and

$$
\begin{aligned}
& \int_{\Gamma}\left|\partial_{\nu} u(t, x)\right|^{2} \mathrm{~d} \Gamma=\sum_{\ell \in\{a, b\}} \int_{0}^{2 \pi}\left|\partial_{r} u(t, \ell, \theta)\right|^{2} d \theta \\
& =2 \pi \sum_{\ell \in\{a, b\}} \sum_{m=1}^{\infty}\left(\left|\sum_{k=1}^{\infty} c_{k, m}^{+} R_{k, m}^{\prime}(\ell) e^{i \frac{\gamma_{m, \alpha, k}}{b} t}\right|^{2}+\left|\sum_{k=1}^{\infty} c_{k, m}^{-} R_{k, m}^{\prime}(\ell) e^{-i \frac{\gamma_{m, \alpha, k}}{b} t}\right|^{2}\right) \\
& +2 \pi \sum_{\ell \in\{a, b\}}\left(\left|\sum_{k=1}^{\infty} c_{k, 0}^{+} R_{k, 0}^{\prime}(\ell) e^{i \frac{\gamma_{0, \alpha, k}}{b} t}+\sum_{k=1}^{\infty} c_{k, 0}^{-} R_{k, 0}^{\prime}(\ell) e^{-i \frac{\gamma_{0, \alpha, k}}{b} t}\right|^{2}\right)
\end{aligned}
$$

Since asymptotic gap is $\frac{1}{b} \frac{\pi}{1-\alpha}=\frac{\pi}{b-a}$ by the formula (5), we may apply Ingham's theorem (more precisely its version due to Haraux, see, e.g., [7]) to deduce from the last equality the existence of $C_{1, M}>0$ such that

$$
\int_{\Gamma}\left|\partial_{\nu} u(t, x)\right|^{2} \mathrm{~d} \Gamma \geq C_{1, M} \sum_{m=0}^{\infty} \sum_{k=1}^{\infty}\left(\left|c_{k, m}^{+}\right|^{2}+\left|c_{k, m}^{-}\right|^{2}\right) \sum_{\ell \in\{a, b\}}\left|R_{k, m}^{\prime}(\ell)\right|^{2}
$$


for all complex sequences $\left(c_{k, m}^{ \pm}\right)$with $c_{k, m}^{ \pm}=0$ whenever $m \geq M$.

Now it remains to prove that there exists another constant $C_{2, M}>0$ such that

$$
\begin{aligned}
& \sum_{\ell \in\{a, b\}}\left|R_{k, m}^{\prime}(\ell)\right|^{2} \\
& \geq C_{2, M}\left(\int_{a}^{b}\left(1+\left|\frac{\gamma_{m, \alpha, k}}{b}\right|^{2}+\frac{m^{2}}{r^{2}}\right)\left|R_{k, m}(r)\right|^{2} r d r+\int_{a}^{b}\left|R_{k, m}^{\prime}(r)\right|^{2} r d r\right)
\end{aligned}
$$

for $m \geq 1$, and a similar inequality for $m=0$.

We adapt an argument used in [7], p. 107. Let $y$ satisfy the Bessel equation

$$
x^{2} y^{\prime \prime}+x y^{\prime}+\left(x^{2}-m^{2}\right) y=0
$$

Let $c>0$. Multiplying the equation by $2 y^{\prime}$ and integrating over $(c a, c b)$ (instead of $(0, c)$ as in [7]), we get

$$
\int_{c a}^{c b}\left(2 x^{2} y^{\prime} y^{\prime \prime}+2 x\left(y^{\prime}\right)^{2}+2\left(x^{2}-m^{2}\right) y y^{\prime}\right) d x=0 .
$$

Integrating by parts the first and third terms, we obtain

$$
\int_{c a}^{c b}\left(-2 x\left(y^{\prime}\right)^{2}+2 x\left(y^{\prime}\right)^{2}+2 x y^{2}\right) d x=\left[x^{2} y^{\prime 2}+\left(x^{2}-m^{2}\right) y^{2}\right]_{c a}^{c b},
$$

so that

$$
2 \int_{c a}^{c b} x y^{2} d x=\left[x^{2} y^{\prime 2}+\left(x^{2}-m^{2}\right) y^{2}\right]_{c a}^{c b}
$$

The change of variable $x=c r$ transforms this into

$$
2 c^{2} \int_{a}^{b} r y^{2}(c r) d r=\left[x^{2} y^{\prime 2}+\left(x^{2}-m^{2}\right) y^{2}\right]_{c a}^{c b} .
$$

Recall that

$$
R_{k, m}(r)=Y_{m}\left(\gamma_{m, \alpha, k}\right) J_{m}\left(\frac{r}{b} \gamma_{m, \alpha, k}\right)-J_{m}\left(\gamma_{m, \alpha, k}\right) Y_{m}\left(\frac{r}{b} \gamma_{m, \alpha, k}\right) .
$$

Now, we define

$$
y_{k, m}(x)=Y_{m}\left(\gamma_{m, \alpha, k}\right) J_{m}(x)-J_{m}\left(\gamma_{m, \alpha, k}\right) Y_{m}(x)
$$

which satisfies (9), and thus we have (10) with $y=y_{k, m}$ and $c=\frac{\gamma_{m, \alpha, k}}{b}$. We have

$$
\begin{aligned}
y(c r)=R_{k, m}(r), y(c a)=R_{k, m}(a)=0, y(c b)=R_{k, m}(b)=0 \\
\\
c y^{\prime}(c r)=R_{k, m}^{\prime}(r)
\end{aligned}
$$


and thus

$$
2 c^{2} \int_{a}^{b} r R_{k, m}(r)^{2} d r=b^{2} R_{k, m}^{\prime}(b)^{2}-a^{2} R_{k, m}^{\prime}(a)^{2}
$$

From (3) we have the relation

$$
r^{2} R_{k, m}^{\prime \prime}(r)+r R_{k, m}^{\prime}(r)-m^{2} R_{k, m}(r)=-c^{2} r^{2} R_{k, m}(r) .
$$

Integrating by parts and using the relations $R_{k, m}(b)=R_{k, m}(a)=0$ we obtain that

$$
\int_{a}^{b} R_{k, m}^{\prime}(r)^{2} r d r=-\int_{a}^{b} r R_{k, m}(r)\left(R_{k, m}^{\prime \prime}(r)+\frac{1}{r} R_{k, m}^{\prime}(r)\right) d r
$$

Using the relation (12) hence we conclude that

$$
\int_{a}^{b} R_{k, m}^{\prime}(r)^{2} r d r=-\int_{a}^{b} r R_{k, m}(r)^{2}\left(-c^{2}+\frac{m^{2}}{r^{2}}\right) d r
$$

Setting

$$
A=\int_{a}^{b}\left(1+c^{2}+\frac{m^{2}}{r^{2}}\right)\left|R_{k, m}(r)\right|^{2} r d r+\int_{a}^{b}\left|R_{k, m}^{\prime}(r)\right|^{2} r d r
$$

we deduce from the above relations that

$$
\begin{aligned}
A & =\left(1+2 c^{2}\right) \int_{a}^{b}\left|R_{k, m}(r)\right|^{2} r d r \\
& =\frac{1+2 c^{2}}{2 c^{2}}\left(b^{2} R_{k, m}^{\prime}(b)^{2}-a^{2} R_{k, m}^{\prime}(a)^{2}\right) .
\end{aligned}
$$

Since

$$
\inf _{k,|m| \leq M}\left|\gamma_{k, \alpha, m}\right|>0
$$

by (5) and the inequalities $\gamma_{k, \alpha, m}>0$, we conclude that

$$
A \leq C_{3, M} R_{k, m}^{\prime}(b)^{2} \leq C_{3, M}\left(R_{k, m}^{\prime}(b)^{2}+R_{k, m}^{\prime}(a)^{2}\right)
$$

for a suitable constant $C_{3, M}>0$. This proves (8) for $m \geq 1$.

For $m=0$, using (13) we deduce from (7) that 


$$
\begin{aligned}
2 \pi \sum_{k=1}^{\infty}\left(\int_{a}^{b}\left(\left|c_{k, 0}^{+}+c_{k, 0}^{-}\right|^{2}+\left|c_{k, 0}^{+}-c_{k, 0}^{-}\right|^{2}\left|\frac{\gamma_{0, \alpha, k}}{b}\right|^{2}\right)\left|R_{k, 0}(r)\right|^{2} r d r\right. \\
\left.+\left|c_{k, 0}^{+}+c_{k, 0}^{-}\right|^{2} \int_{a}^{b}\left|R_{k, 0}^{\prime}(r)\right|^{2} r d r\right) \\
=2 \pi \sum_{k=1}^{\infty}\left(\int_{a}^{b}\left(\left|c_{k, 0}^{+}+c_{k, 0}^{-}\right|^{2}+\left|c_{k, 0}^{+}-c_{k, 0}^{-}\right|^{2}\left|\frac{\gamma_{0, \alpha, k}}{b}\right|^{2}\right)\left|R_{k, 0}(r)\right|^{2} r d r\right. \\
\left.+\left|c_{k, 0}^{+}+c_{k, 0}^{-}\right|^{2}\left|\frac{\gamma_{0, \alpha, k}}{b}\right|^{2} \int_{a}^{b}\left|R_{k, 0}(r)\right|^{2} r d r\right) \\
\asymp \sum_{k=1}^{\infty}\left(\left|c_{k, 0}^{+}\right|^{2}+\left|c_{k, 0}^{-}\right|^{2}\right)\left|\frac{\gamma_{0, \alpha, k}}{b}\right|^{2} \int_{a}^{b}\left|R_{k, 0}(r)\right|^{2} r d r
\end{aligned}
$$

and we conclude as before.

\section{References}

1. M. Abramowitz, I.A. Stegun (EDS.), Handbook of mathematical functions, Dover, New York, 1972.

2. C.Bardos, G. Lebeau, J. Rauch, Sharp suffcient conditions for the observation, control and stabilization of waves from the boundary, SIAM J. Control Optim. 30 (1992), 1024-1065.

3. http://cose.math.bas.bg/webMathematica/webComputing/BesselZeros.jsp

4. H.P.W. Gottlieb, Eigenvalues of the Laplacian with Neumann boundary conditions, J. Austral. Math.Soc. Ser. B 24 (1983), 435-438.

5. A. Haraux, Séries lacunaires et contrôle semi-interne des vibrations d'une plaque rectangulaire, J. Math. Pures Appl. 68 (1989), 457-465.

6. A. E. Ingham, Some trigonometrical inequalities with applications in the theory of series, Math. Z. 41 (1936), 367-379.

7. V. Komornik, P. Loreti, Fourier Series in Control Theory, 2005, Springer.

8. Lebedev,Skalskaya,Uflyand Worked problems in applied mathematics, Problem 121. 